wenigstens glaube ich diesem eine grössere Farbenintensität als dem Crocus zuschreiben und zu analogen Zwecken empfehlen zu dürfen.

\title{
Ueber Pepsin.
}

Von E. Heintz, Apotheker in Duisburg.

In letzter Zeit sind unter dem Namen Pepsin wieder verschiedene Mischungen in den Handel gekommen und verschiedene Vorschriften zur Bereitung derselben gegeben worden. In dem Lehrbuch der „Physiologischen Chemie von v. Gorup-Besanez 1867" steht Seite 454:

"Alle die Stoffe (nemlich die als reines Pepsin bezeichneten) sind als chemisch reine Substanzen nicht anzusehen und nur in sofern von physiologischem Interesse, als sie das wirksame Verdaungsferment in mehr oder weniger concentrirtem Zustande enthalten."

Man lese in diesem vorzüglichen Werke hieruber ein Weiteres. Daselbst ist auch angedeutet, dass die Dialyse möglicherweise ein reines Pepsin herzustellen vermöge. Es wird zugegeben, dass die Pepsin haltenden Lösungen Albumin, Leim und leimgebende Stoffe nicht bloss lösen, sondern verdauen können, sobald keine, diese Verdaung störenden Substanzen vorhanden sind. Als solche sind auch Alkohol, Fett und grosse Mengen Speichel (unten die Versuche) aufgeführt. Also reines Pepsin kenmen wir nicht.

E. Scheffer in Louisville giebt im Archiv (Angust 1870 S. 164) eine Vorschrift, die sich der schon früher von Hager (Centralhalle) gegebenen anschliesst. Scheffer wendet zur Lösung nur Glycerin und Wasser an, Hager mischt noch $10^{\circ}$ Alkohol haltenden Rheinwein zu, Scheffer will alle Alkohol haltenden Flïssigkeiten vermieden wissen und möchte ich mich (nach den unten angegebenen Versuchen) Scheffer's Vorschlag durchaus anschliessen. Hager war eine Nachricht zugegangen, dass Sch effer'sche Pepsinlösung abscheulich schmecke. Dass ein Zusatz von Wein den Pepsin- 
Geschmack (ich sage der Kürze wegen Pepsin, wie reines Pepsin schmeckt, wissen wir ja nicht) vermindert, resp. verbessert, ist ersichtlich. Zwischen Schering's' Lösung von Lamatsch, Berliner oder nach Seheffer bereiteter Pepsinlösung war mir kein Unterschied bemerkbar, der das eine als abscheulich schmeckend bezeichnen könnte. Scheffer's Pepsin schmeckt eben stärker nach Pepsin. Doch das ist Geschmacksache. In dem genannten Werke giebt Gor up-Besanez an, dass schon zur Peptonisirung benutzte Pepsinlösung, durch Dialyse von Pepton getrennt, wieder peptonisirend wirken könne. Danach wäre der Gehalt an Pepsin nicht allein maassgebend und also alle haltbaren Präparate ohne störende Beimischungen anwendbar. Dennoch schlage ich vor, nach Ha g er's oder S c h e ffer's Vorschrift bereitete Mischung, (erstere ohne Wein und bei beiden mit mehr Wasser) bis zu dem spec. Gewicht von 1,12 bei $15^{\circ} \mathrm{C}$. einzudampfen. Das Eindampfen darf natürlich bei höchstens $35^{\circ} \mathrm{C}$. stattfinden und geht darum ziemlich langsam. Aber ein so eingedampftes Pepsin hält sich ausgezeichnet und kann, um es noch haltbarer zu machen, mit gleichen Gewichtstheilen Glycerin vermischt werden. Es kommt dann ungefähr auf sein ursprüngliches Volumen. Scheffer's Pepsin nemlich schimmelt, Hager's Pepsin würde dies ohne Weinzusatz auch thun. Ich will schon hier bemerken, dass auf die angegebene Weise bereitetes Pepsin, mit und ohne nachträglichen Glycerinzusatz (mit Wasser verdünnt) vollständig peptonisirend wirkte, obgleich die Pepsinlösung (nach Scheffer) vor dem Eindampfen etwas verschimmelt war. Das Pepsin, vom Schwein gewonnen, neigte mehr zum Schimmeln, wie das rom Rind. Die wiederholt verschimmelten und von dem Schimmel abfiltrirten Lösungen zeigten immer weniger Pepsingeschmack, während ihre peptonisirende Wirkung nicht zu leiden schien. Bei dem Eindampfen geht auch etwas Salzsäure verloren, man setzt daher $1 / 10$ des ursprünglichen Gewichtes Salzsäure nach dem Eindampfen zu. Bekannt ist es ja, dass bei zu hoher Temperatur z. B. im Dampfbade eingedicktes Pepsin seine peptonisirende Kraft vollständig 
verliert, wieich ebenfalls durch Versuche erkannt habe. Merkwürdig ist auch, dass. Ol. menth. pip.das Schimmeln unterdrückt. Verschimmelte Pepsinlösung wurde filtrirt und das Filtrat, je $20 \mathrm{Gr}$. mit 1 Tropfen 0l. menth. pip. opt. versetzt, die andere Hälfte ohne Ol. m. in verkorkten Flaschen hingestellt. Die letztere war schon den andern Tag verschimmelt, die erstere zeigt heute nach 25 Tagen auf dem Filter noch keine Spur Schimmel. Vielleicht bewirken andere ätherische Oele dasselbe? Jedenfalls giebt Ol. menth. p. ein ganz passendes weiteres Conservirungs Mittel ab. Frische Pepsinlösung 25,0, 0l. menth. p. 1 Tropfen, Salzsäure 5 Tropfen auf 2,0 coagulirtes Eiweiss bewirkten ziemlich rasch Lösung des Eiweisses. Das Filtrat coagulirte durch Kochen, das Filtrat hiervon gab mit $\mathrm{NO}^{5}$, keine Reaction, wohl aber mit Gerbsäure.

$\mathrm{Ob}$ es nöthig ist, dass die Pepsinlösung eine einfache rasche Lösung des coagulirten Eiweisses bewirke, oder dasselbe in eine der verschiedenen Peptonarten überführe, darüber habe ich kein Urtheil und nach den von mir angestellten wi $\mathrm{d}$ e rholten Versuchen zeigte sich bei ähnlichen Versuchen keine Gleichheit. Frische Pepsinlösung scheint rascher zu wirken, als solche, die einige Zeit gestanden hat und wirkt die frische dann theils nur lösend theils peptonisirend auf coagulirtes Eiweiss. Frisch gewonnene Pepsinlösung und sofort bei $35^{\circ}$ zum Eindampfen angesetzte und concentrirte wirkte ebenso rasch lösend, wie nicht eingedampfte. Kochen schied Eiw. ab, das Filtrat hiervon gab mit $\mathrm{NO}^{5}$ noch eine Reaction.

Zum Nachweis des Pepton's nahm ich das Nichtcoaguiren des Filtrats der Lösung durch Kochen an, aber dies Filtrat musste mit $\mathrm{NO}^{5}$ eine Reaction auf Eiweiss geben. Bei einfacher Lösung schied sich im Filtrat durch Kochen schon das Eiweiss ab. Die empfindliche Probe nit Gerbsäure konnte nicht angewandt werden, weil reine Pepsinlösung, die durch Kochen coagulirt war, in dem hiervon gewonnenen Filtrat noch mit Gerbsäure eine Reaction gab (Schleim?), $\mathrm{NO}^{5}$ reagirte dann nicht mehr. 
Da pflanzliches Eiweiss sich nach Angabe von v. GorupBesanez, ebenso gegen Pepsin verhält, wie thierisches, so muss es gleich sein, ob das Pepsin von Rind, oder von Schwein etc. gewonnen wird. Für Straussenmagen müssten wir ja eine Straussenzucht haben, um nennenswerthe Mengen Pepsin zu gewinnen.

Die flüssigen, ans Schwein - und Rindsmagen gewonnenen Pepsinlösungen scheinen übrigens schon Eiweiss zu enthalten. Sie bräunen alkal. Wismnthlösung und zeigen in der alkal. Kupferoxydlösung die schön violette Färbung (Tyrosin?). In derselben Lösung zeigt sich anch die schön blaue Färbung, durch Glycerin bewirkt.

Lamatsch's Pepsin bräunt die Wismuthlösung kaum, Berliner gar nicht.

Sämmtliche angeführte Pepsine, die ich in Händen hatte, enthalten keinen Zucker und führen auch Stärkekleister nicht in Zucker über. Zum Versuch kamen:

Lamatsch's Pepsin, trocknes Pulver, frei von Stärke. In Wasser wenig löslich, Lösung schwach sauer. Kalilauge nahm etwas mehr auf. Es ist dies wahrscheinlich die vorsichtig abgekrazte Schleimhaut des Rindsmagens, bei niedriger Temperatur eingetrocknet.

Berliner, sogenantes lösliches Pepsin. Sehr hygroskopisches bräunliches Pulver. Leicht und vollständig löslich in Wasser, Lösung entschieden sauer.

Pepsin rom Schweinsmagen nach Scheffer.

Pepsin rom Rindsmagen nach Scheffer.

Einige Versuche folgen noch, die gemacht wurden, um eine Controle über die Wirkung derjenigen Stoffe zu haben, die bei dem Verdauungsprocesse doch mitwirken. Ausgenommen natürlich die Versuche mit Wein und Ol, menth. pip.

1) Salzsäure $20 \mathrm{Tr}$. 20,0 Wasser

Lösung nur eine Spur.

1,0 coagulirt. Eiweiss $\int$ Kochen keine, $\mathrm{NO}^{5}$ eine Reaction.

2) Ebenso, aber statt Wasser Rheinwein

Keine Lösung, auch gar keine Reaction. 
3) Ebenso wie 1 u. $1 \mathrm{Tr}$. Lösung eine Spur.

Ol. menth. p. $\quad$ Kochen keine, $\mathrm{NO}^{5}$ eine Reaction.

$\left.\begin{array}{l}\text { 4) Wie 1, aber statt } \\ \text { Wasser Malaga }\end{array}\right\}$ Wic 2 .

5) Salzsäure $20 \mathrm{Tr}$.

12,0 Spoichel

12,0 Wasser

Coag. Eiw. 2,0

Lösung unbedentend.

Kochen keine, $\mathrm{NO}^{5}$ eine Reaction.

6) Salzsäurc 5 Tr.

7,0 Speichel 15,0 Pepss. Lös. v. Rind

Coag. Eiw. 2,0

7) Kochsalz 0,5

Wasser 20,0

Coag. Eiw. 2,0

Rasche Lösung.

Kochen keine, $\mathrm{NO}^{5}$ eine Reaction. Ein solches Verhältniss in Bezug auf Speichel kann also nicht als "grosse Menge" bezeichnet weriden und wirkt nicht störend.

\section{Das hicrvon gewonnene and abgewaschene}

8) Eiweiss

Kochsalz 0,5

Salzsïure 20 Tr.

Keine weitcre Lösung und keine Reaction.

Zu dieser Nlischme wurde nun

9) Pepsinlös. v. Rind 25,0 gesetzt. Das Eiweiss löste sich zu circa $2 / 3$ und gab durch Kochen eine Reaction.

10) Berliner Pepsin 0,3

Salzsäure $15 \mathrm{Tr}$.

Wenig gelöst.

Wasser 30,0

Coag. Eiw. 2,0

Kochen keinc, $\mathrm{NO}^{5}$ eine Reaction.

11) Wie 10 , aber

Wasser 25,0

Rheinwein 25,0

12) Wie 10, statt

Wasser Rheinwoin

Wie 10.

13) Wie 10 , statt

Wasser Malaga Wein $\{$ Keine Lösung. 
14) Lamatsch' Pepsin 0,3

Salzsäure 20 Tr.

Rasche Lösung.

C. Eiw. 2,0

Wasser 30,0

Kochen keine, NO ${ }^{5}$ Reaction.

Dieselben Mischungen wie 11, 12 und 13 statt Berliner, aber Lamatsch' Pepsin gaben nach 11 und 12 geringe Lösungen, nach 13 mit Malaga keine Lösung.

15) Pepsinlös. v. Schwein Rasche Lösung.

C. Eiw. 1,0

Salzsäure $5 \mathrm{Tr}$. 15,0 Kochen starke Abscheidung, das

16) Ebenso und Zusatz von Rheinwein 15,0 Filtrat hiervon noch Reaction mit $\mathrm{NO}^{5}$.

\section{Lösung gut die Hälfte.}

Kochen Reaction; Filtrat hiervon mit $\mathrm{NO}^{5}$ keine Reaction.

18) Pepsinlös. v. Rind 15,0

C. Eiw. 1,0

Salzsäure 5 Tr.

19) Wie 18 und Zusatz von

Rheinwein 15,0

Verhalten bei rascher Lösung wie 15 und 16, jedoch durch Kochen viel weniger Abscheidung, stärker mit $\mathrm{NO}^{5}$.

Es wurde schon oben erwähnt, dass sich die bis zum spec. Gew. von 1,12 bei $35^{\circ}$ eingedampften und wieder mit Wasser verdünnten Pepsinlösungen ebenso kräftig lösend verhielten, wie die nicht eingedampften. Diese Letzteren zeigten bei frischer Bereitung ein spec. Gew. von 1,04 bis 1,06 bei $13^{\circ} \mathrm{C}$. Auch die eingedampften, mit gleichen Theilen Glycerin vermischten und mit $\mathrm{W}$ asser etwas verdünnten Lösungen zeigten sich kräftig lösend. Auffallend ist, dass Pepsinlösung, die doch durch Kochen coagulirt, und Salzsäure dieses Coagulum nicht auflöst, bei einigen Versuchen diese Eigenschaft verloren hatte. Auch schien es mir, als ob in dem coagulirten Eiweiss immer ein Theil löslicher war, wie ein anderer.

$\mathrm{Zu}$ allen Versuchen wurde destillirtes Glycerin und dest. Wasser angewandt. Ein Schütteln der Mischungen fand nicht statt. Constante Temperaturen konnten, besonders bei Nacht, nicht inne gehalten werden. Bei schlechter Lösung blieben die Mischungen länger stehen. 
Ein Versuch wurde noch gemacht. Ein 1 Meter und ein $1 / 2$ Meter langer Darm vom Schwein, dicht am Magen, wurde der Länge nach aufgeschnitten, der von Galle gelbliche Speisebrei vorsichtig abgewaschen und nun der auf dem Darm sitzende Schleim abgeschabt. Dieser Schleim wurde nach Sch effer's Vorschrift mit Glycerin, Salzsäure und Wasser behandelt und hiervon 15,0 Grm., Coag. Eiw. 1,0, Salzsäure 7 Tr. und eine ebensolche Mischung mit noch 15,0 Rheinwein angesetzt. Es fand eine ganz geringe Lösung statt, die durch Kochen nicht, aber mit $\mathrm{NO}^{5}$ reagirte. Eine bis zum spec. Gew. bei $35^{\circ}$ eingedampfte Lösung des Schleimes gab mit coag. Eiweiss ganz wenige Lösung, rasch Reaction mit NO5. Doch giebt die Schleimlösung für sich schon Reaction mit $\mathrm{NO}^{5}$ und coagulirt durch Kochen nicht.

Diese Schleimlösung führt Stärkekleister nicht in Zucker über. Ferner wurde Glycerin 10,0, dest. Wasser 10,0, Salzsäure $10 \mathrm{Tr}$, coag. Eiw. 1,0 angesetzt. Durchaus keine Einwirkung, selbst Gerbsäure gab keine Reaction.

Weder die Schleimlösung des Darms, noch die fliissigen Pepsinlösungen drehen die Polarisationsebene. Dagegen dreht die Eiweisslösung in Pepsinlösung (nach Scheffer) die Polarisationsebene nach Iinks. In neuerer Zeit wird bekanntlich die Salzsäure dem Pepsin zugesetzt. Aeltere Aerzte verschreiben das Pepsin in Pulver vielfach mit 2 fach kohlensaurem Natron. Alkalien und kohlensaure Alkalien wirken bekanntlich lösend auf Eiweiss.

Nach obigen Versuchen, die wohl nur eine Wiederholung bekannter Sachen sind, glaube ich mich zu dom Schlusse berechtigt, bei der Bereitung von flüssigen Pepsinmischungen alle Spirituosen und Weine, besonders starke Weine fern zu halten. Wein ist überdies ein nnsicherer Artikel, während Glycerin leicht auf seine Güte untersucht werden kann.

Ein Patient, der Pepsin nimmt, müsste dies gleich vor und nach der Mahlzeit nehmen und allenfalls nicht viel Fett geniessen. Sollen doch starke Weine getrunken werden, so müsste dies 2 Stunden vor einer Mahlzeit geschehen. Was 
Scheffer von der verdauenden Wirkung der Spirituosen und starken Weine sagt, so kann sich dies nicht auf Eiweiss, sondern nur auf Fette beziehen, die ja bekanntlich mit Alkohol eine Art Emulsion bilden.

\title{
Auflöslichkeit des Leims in Glycerin.
}

\author{
Von John M. Maiseh.*)
}

Die Resultate einer Untersuchung dieser Löslichkeit lassen sich in folgende Sätze zusammenfassen:

1) Leim ist in einer reichlichen Menge von Glycerin bei gewöhnlicher Temperatur löslich.

2) Er ist fuir Glycerin durchdringlich, langsam bei gewöhnlicher, rascher bei erhöhter Temperatur.

3) Leim, in Folge einer Absorption von Wasser aufgeschwellt, bleibt unter Glycerin scheinbar unveründert, aber das Letztere entzieht dem geschwellten Leime Wasser, in Folge dessen dieser zusammenschrumpft.

4) Bei fortgesetzter Digestion löst sich der Leim vollständig im Glycerin und die Lösung gelatinirt beim Abkïhlen.

5) Die Lösung des Leims in Glycerin wird durch vorhergegangene Maceration in demselben beschleunigt, ebenso durch Temperaturerhöhung (ohne Zweifel auch bei Erhöhung des Druckes).

6) Leim, von Wasser vollständig durchdrungen, löst sich in heissem Glycerin eben so schnell als in heissem Wasser.

Es scheint mir, dass dieses Verhalten des Glycerins, mit Leim eine Gallerte von jeder wünschbaren Consistenz zu bilden, in der Medicin angewendet werden könne, um Vehikel für alle Arzneien von unangenehmem Geschmack zu liefern.

*) Als Separatabdruck aus ,the American. Journ. of Pharmacy," Fobr. 1871 rom Hrn. Verf. eingesandt. (Im Auszuge).

H. $\boldsymbol{L}$. 